\title{
Prevalence and classification of high antimicrobial resistant Staphylococcus aureus in wastewater eluted from poultry slaughterhouse
}

\author{
${ }^{1}$ Abidatul, A.A., ${ }^{1}$ Nur Farhanah, N.M.J., ${ }^{1}$ Noramirah, R., ${ }^{1}$ Ling, S., ${ }^{1 * N e w}$ C.Y. and \\ ${ }^{1,2}$ Son, R. \\ ${ }^{1}$ Department of Food Science, Faculty of Food Science and Technology, Universiti Putra Malaysia, 43400 \\ UPM Serdang, Selangor Darul Ehsan, Malaysia \\ ${ }^{2}$ Food Safety and Food Integrity, Institute of Tropical Agriculture, Universiti Putra Malaysia, 43400 UPM \\ Serdang, Selangor Darul Ehsan, Malaysia
}

\section{Article history:}

Received: 30 November 2017

Received in revised form: 19

December 2017

Accepted: 29 December 2017

Available Online: 31

December 2017

\section{Keywords:}

Staphylococcus aureus,

MRSA,

Antimicrobial resistance

wastewater,

poultry slaughterhouse

\section{DOI:}

https://doi.org/10.26656/fr.2017.2(2).001

\begin{abstract}
The continued and increasing development of antimicrobial resistant bacteria among the foodborne pathogens had caused worldwide to be alarmed. Being the earliest to develop antimicrobial resistance, Staphylococcus aureus is constantly monitored for any new resistance development. The resistance development is often linked to wastewater and the treatment plants where the pressure of antibiotic is the highest. Hence, this study investigated on the prevalence of high antimicrobial resistant $S$. aureus in the wastewater eluted from a poultry slaughterhouse. A total of thirty wastewater samples were collected from a poultry slaughterhouse in Semenyih, Selangor. Most probable number (MPN)plating method was employed to enumerate the $S$. aureus count in the wastewater. The results indicated that $S$. aureus was highly present whereby all samples $(100 \%)$ were positive and the concentration ranged between $11-2.1 \times 10^{4} \mathrm{MPN} / \mathrm{ml}$. Isolated $S$. aureus strains were screened for their antimicrobial susceptibility using the Kirby-Bauer Disk Diffusion Test method to classify their antimicrobial resistance eleven antibiotics. The MAR index measured was between 0.18 and 0.91 , inferring that the strains are highly antimicrobial resistance. All S. aureus strains were $100 \%$ resistant to ampicillin $(25 \mu \mathrm{g})$ and cefazolin $(30 \mu \mathrm{g}) .94 .1 \%$ of the strains were resistant to penicillin $(10 \mu \mathrm{g})$ which phenotypically indicated these strains are Methicillin-resistant S. aureus (MRSA). Notably, $17.6 \%$ of the strains developed resistance to vancomycin and was categorized as Vancomycin-resistant $S$. aureus (VRSA). There is a need to take drastic preventive measures to control the resistance development in $S$. aureus to conserve public health.
\end{abstract}

\section{Introduction}

Poultry is one of the foods most consumed by Malaysian ( $(92.80 \%$ of 24.7 million population) The World Bank, 2017) according to Ministry of Health Malaysia (2006). To produce poultry or any other food animal-based origin, proper handling and processing are important in order to have a good quality product and to secure the safety of the consumer. There are many elements that are of major concerns during the livestock operation and when processing poultry as the intensive process management results in sharing both commensal flora and pathogens (Landers et al., 2012) may result in infection, particularly antibiotic resistant bacterial infection in humans when consuming poultry.

The use of antibiotic in livestock has exposed humans to higher risk of antibiotic resistance. Antibiotic resistant bacteria have become the biggest threats, creating outbreaks and increasing mortality rates. Berglund (2014) mentioned that in the European Union, there are over 25,000 deaths every year caused by antibiotic resistant bacteria. While Centers of Disease Control and Prevention (CDC) (2017) reported that at least 23,000 deaths were caused by antibiotic resistance in the United States. In South-East Asia region, there are no systematic reports compiled to illustrate the antibiotic resistance situation. However, information and data available for selected organism developing resistance were summarized by Bhatta and Narain (2010), indicating the widespread of antibiotic resistance bacteria worldwide.

The resistance of microorganism towards a drug that used to be effective before is defined as antibiotic/ 
antimicrobial resistance (AMR). These microorganisms are able to survive when antimicrobial medicines, such as antibiotics, antifungals, antivirals, and antimalarial comes to attack or fight against them. Antibiotics are commonly placed in food animals for therapeutic use, prophylactic use, and subtherapeutic use (Landers et al., 2012). However, the overuse and misuse of antibiotics (Wright, 2010; Berglund, 2014) due to the widespread adoption of antibiotic use in food animals (Sarmah et al., 2006) had exacerbated the matter. This had caused the decreased efficiency of the antibiotics and reduced the therapeutic options (Appelbaum, 2012; Berglund, 2014).

The first antibiotic resistant bacteria identified was penicillin-resistant Staphylococcus in 1940 when penicillin was commercialized (CDC, 2017). Staphylococcus aureus, a type of bacteria that is commensal on the human skin can cause serious infection in healthcare settings. Furthermore, $S$. aureus was known to be resistant to be several types of antibiotic such as methicillin, vancomycin, linezolid and ceftaroline. Methicillin-resistant S. aureus (MRSA) was identified in 1962, two years after methicillin was introduced. According to the Antibiotic Resistance Threats in the United States (2013), MRSA was labelled as a serious threat level. CDC (2013) reported that there are 80, 461 severe MRSA infections per year and 11, 285 deaths per year were caused by MRSA infection.

Nevertheless, the spread of MRSA among the general population - those not recently associated in a healthcare setting had been reported and increasing (CDC, 2013). The possibility of the general population contracting MRSA maybe from the food from animals that are fed with antibiotics, environment or direct human-animal contact. Thus, this research answers the purpose to study on the spread of antibiotic resistant bacteria, in particular $S$. aureus, to humans through the environment in a real scenario. The objectives of this study were to isolate, identify and enumerate $S$. aureus from the wastewater eluted from a poultry slaughterhouse and also, to develop the antibiogram profiles of the isolated $S$. aureus. Antibiograms profile is used to relate the respond of the $S$. aureus with the antibiotics. Improper practices and handling of wastewater is a frequent issue, and a part of contributor to the spread of antibiotic resistance bacteria (Baddour et al., 2016).

\section{Materials and methods}

\subsection{MPN-Plating}

A total of thirty samples were collected at a poultry slaughterhouse located at Semenyih, Selangor. The samples were collected in batches on different days. The wastewater was collected before it goes to the drain by using a sterile funnel and $500 \mathrm{ml}$ sized empty sterile drinking water bottle. The samples were brought back to the laboratory immediately for analysis.

Ten $\mathrm{ml}$ of the collected effluent was added with 90 $\mathrm{ml}$ of Tryptic Soy Broth (TSB) to dilute the effluent to $10^{-1}$ dilution. The sample was then stomached for 30 seconds using the stomacher machine and then proceeded to quantification using MPN method according to Bennett and Lancette (2016). The dilution was serially diluted with $9 \mathrm{ml}$ TSB to make $10^{-2}, 10^{-3}, 10^{-}$ ${ }^{4}$, and $10^{-5}$ dilution. Then, $1 \mathrm{~mL}$ of each dilution was transferred into triplicate tubes containing $9 \mathrm{ml}$ of TSB with $10 \%$ sodium chloride, $\mathrm{NaCl}$, for incubation. The triplicate tubes were incubated at $37^{\circ} \mathrm{C}$ for 48 hours. After incubation, the turbidity of all tubes was examined for presence or absence of growth (Sutton, 2010). The positive tubes were streaked on the Mannitol Salt Agar (MSA) plate, inverted followed by incubation at $37^{\circ} \mathrm{C}$ overnight. Plates were examined for presumptive $S$. aureus colonies and the growth pattern was scored against the MPN table (Blodgett, 2010). Identified presumptive $S$. aureus colonies were stored in agar slants for temporary storage. The presumptive $S$. aureus colonies were confirmed via Polymerase Chain Reaction (PCR) targeting the $n u c$ gene at 270 bp amplicons following the method described by Brakstad et al. (1992).

\subsection{Antibiotic susceptibility testing}

Antibiotic susceptibility testing was performed following the disk diffusion test as described by Bauer et al. (1966) with modification. Antibiotics that were being tested were amoxicillin $(25 \mu \mathrm{g})$, ampicillin $(25 \mu \mathrm{g})$, cefazolin $(30 \mu \mathrm{g})$, ciprofloxacin $(10 \mu \mathrm{g})$, erythromycin $(15 \mu \mathrm{g})$, kanamycin $(30 \mu \mathrm{g})$, norfloxacin $(10 \mu \mathrm{g})$, penicillin $(10 \mu \mathrm{g})$, tetracycline $(10 \mu \mathrm{g})$, trimethoprim (5 $\mu \mathrm{g})$ and vancomycin $(30 \mu \mathrm{g})$. All antibiotics were purchased from Oxoid, United Kingdom. Positives confirmed $S$. aureus isolates were inoculated in $9 \mathrm{ml}$ Mueller-Hinton broth (MHB) and incubated at $37^{\circ} \mathrm{C}$ for 18 to 24 hours. The suspensions were then swabbed onto Mueller-Hinton agar (MHA) using a sterile cotton swab and were air dried. Antibiotic discs were arranged by using sterile forceps on the swabbed plates. The plates were then inverted and incubated for 24 hours at $37^{\circ} \mathrm{C}$. Diameter of the present hollow zone was measured and compared with M100S Performance Standards for Antimicrobial Susceptibility Testing, $26^{\text {th }}$ edition from Clinical and Laboratory Standards Institute (CLSI) (2016)

Multiple antibiotic resistant (MAR) index was calculated in this study as the ratio to the number of 
antibiotics to which the strain resistant to. The number of antibiotics that resistant by the bacteria was divided by the total number of antibiotics.

\section{Results and discussion}

Among the thirty wastewater samples eluted from the poultry slaughterhouse, all of them were positive for S. aureus $(100 \%)$ and the MPN range was between 11 to $21000 \mathrm{MPN} / \mathrm{ml}$. The source of $S$. aureus could be from the slaughterhouse, livestock and the food handlers. The presence of $S$. aureus is inevitable as $S$. aureus can be found in animals especially in the skin, mucous membrane of the upper respiratory tract, lower urogenital tract and digestive tract (Kluytmans, 2010; Kumar et al., 2015). Additionally, a wide range of animals and birds carries S. aureus (Food Standard Agency, 2017). These $S$. aureus strains are identified as the poultry biotypes. On the other hand, the human biotypes $S$. aureus whereby the strains originate from humans also has the possibility to contaminate the raw chicken during process handling and the slaughterhouse by the workers when food safety measures are not taken. Approximately, $30 \%$ of the human population are $S$. aureus carriers and Arai et al. (2004) also stated that $S$. aureus and enterotoxigenic $S$. aureus strains were frequently detected on the hands of workers in a poultryprocessing plant. In addition, Losito et al. (2005) stressed that worker's hand before slaughtering and defeathering equipment were the main crosscontamination point. This implied that the chickens were not only infected with the $S$. aureus poultry biotypes but could also be infected with the $S$. aureus human biotypes in which the $S$. aureus was shed off during the poultry processing and cleaning of the slaughterhouse to be present in the wastewater.

The danger of having this microorganism in the wastewater is that there is a risk of increased exposure to the environment. What worsens the matter is that the wastewater eluted from the poultry slaughterhouse was not treated. According to the United Nations (2017), it was reported that $80 \%$ of the global untreated wastewater is released and the consequences are alarming. Water pollution increases having its body contaminated with bacteria, nitrates, solvents, and phosphates (DW, 2017), and as the pollution travels, it creates issues in the environment and human health are affected. It is reported that antimicrobial resistance is on the rise due to the excessive and uncontrollable use of antibiotics, especially in animal husbandries. There is sufficient epidemiologic evidence of an association between antibiotic use in food animals and antibiotic resistance in humans as suggested by Landers et al. (2012). Animals are commonly fed with food animals incorporated with antibiotics. Microorganisms infecting the livestock are constantly pressured with high concentrations of antibiotics and subsequently gaining more antibiotic resistance. Moreover, excessive antibiotics are also released into the wastewater through animals' excretion where it will be collected in the treatment plants, creating reservoirs for antibiotic resistance. This is also supported by Rowan (2011) that the untreated wastewater has high antibiotics that can cause toxic degradation formation that can be harmful to the environment and public health.

Aforementioned, $S$. aureus was the first pathogen that developed resistance towards antibiotics (New et al., 2016). To study the antibiotic resistance pattern, the antibiogram profile of the $S$. aureus isolates was visualized in Figure 1. From Figure 1, it can be seen that the $S$. aureus isolates showed the highest resistance $(100 \%)$ towards ampicillin and cefazolin which implied that these antibiotics are no longer effective against $S$. aureus. The study conducted by New et al. (2016) also reported that cefazolin is no longer effective towards the S. aureus strains.

S. aureus can be categorized as MRSA if the microorganism is resistant towards beta-lactam antibiotics (New et al., 2016). MRSA, as described by Abdalrahman et al. (2015) is resistant to penicillin and other $\beta$-lactam antibiotics by protecting the mecA gene encoding the penicillin-binding protein $2 \mathrm{a}$ (PBP2a). Amoxicillin, ampicillin, oxacillin, and penicillin are examples of $\beta$-lactam antibiotics. From this study, 94.1\% of the isolated $S$. aureus strains were resistant towards penicillin whereas $82.4 \%$ of the isolates were resistant to amoxicillin (Figure 1). This indicated that well above $80 \%$ of the $S$. aureus isolates falls under the group of MRSA. According to CDC (2016), strains that cause healthcare-associated infections (also known as hospitalacquired MRSA) are often resistant to other commonly used antimicrobial agents, including erythromycin, clindamycin, fluoroquinolones (i.e. ciprofloxacin and norfloxacin in this study) and tetracycline.

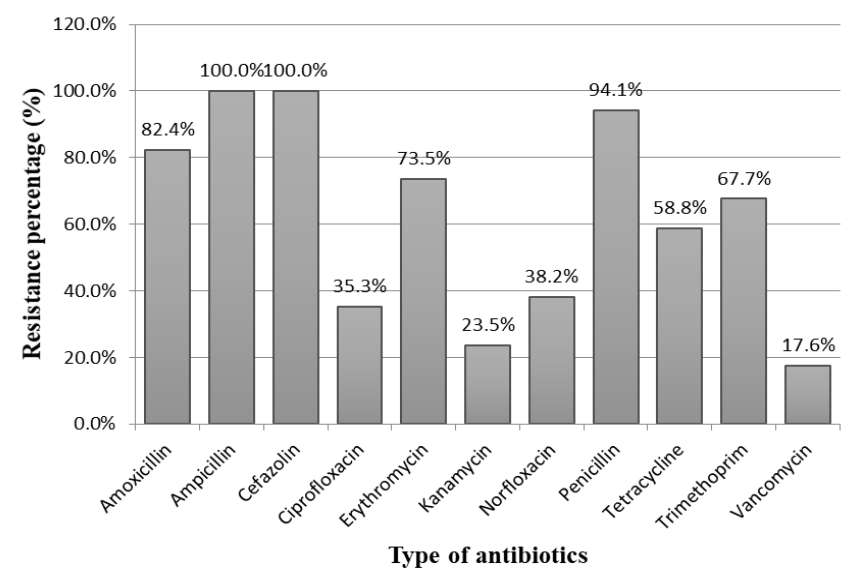

Figure 1. Antibiogram profile of isolated Staphylococcus aureus from wastewater 
Based on Figure 1, the results showed the isolated $S$. aureus strains were $73.5 \%$ resistant to erythromycin; $35.3 \%$ resistant to ciprofloxacin; $38.2 \%$ resistant to norfloxacin and; $58.8 \%$ resistant to tetracycline which implied that the isolates could be a mixture of hospitalacquired MRSA and community-acquired MRSA. In contrast to hospital acquired MRSA, communityacquired MRSA often affects the community and characterized to be resistant to beta-lactam antibiotics and erythromycin as well as may be resistant to fluoroquinolones (CDC, 2016). In this study, the antibiogram profile of the isolates was able to categorize $29.4 \%$ of the isolates as hospital acquired MRSA and community-acquired MRSA respectively.

Community-acquired MRSA has become a major global challenge as it was found that this is a factor of rapid spreading of MRSA (Ike et al., 2016). According to Food Standard Agency (2017), the direct contact between human with individuals infected with MRSA is the main transmission route of hospital-acquired MRSA and community-acquired MRSA whereas, the direct contact with infected food-producing animals, companion animals (including horses), wild birds, watercourses and through contaminated dust and aerosols can cause transmission of livestock acquired MRSA to public. Additionally, the spread of antimicrobial resistant bacteria commonly occurs between humans and poultry (Rogers and Haines, 2005).

Vancomycin-resistant S. aureus (VRSA) is emerging rapidly in the environment since 1996 and, this has been highlighted in studies conducted by Lowy (2003) and Berglund (2014). In some cases, these strains might be MRSA and they are fully resistant to vancomycin. Vancomycin used to be the selected antibiotic to treat severe infection of $S$. aureus. However, the reduced efficacy of the drug had led to greater issues in suppressing the bacteria. Notably, there are only $17.6 \%$ of the isolates that were resistant to vancomycin, suggesting VRSA have not been spread widely. In comparison with the study conducted by New et al. (2016) which reported $66.7 \%$ of the $S$. aureus isolates were categorized as VRSA. VRSA should not be taken lightly and should be monitored. Kanamycin, an antibiotic used for treatment against variety infection was found to be effective against the isolated $S$. aureus strains as the resistance of the strains were recorded at $23.5 \%$. On the other hand, $67.7 \%$ of the isolated $S$. aureus strains showed resistivity to trimethoprim.

The MAR index obtained in this study ranged between 0.18 and 0.91. Rahman et al. (2015) stated that there is a high risk of contamination of several antibiotics used in the environment of the isolate originated if MAR index obtained is more than 0.2. The least number of antibiotics that $S$. aureus strain was resistant was two out of eleven antibiotics. The highest index referred to the strains that were resistant to ten antibiotics. There are $5.9 \%$ isolates resistant to two antibiotics; $8.8 \%$ resistant to four antibiotics; $8.8 \%$ resistant to five antibiotics; $11.8 \%$ resistant to six antibiotics; $20.6 \%$ resistant to seven antibiotics; $20.6 \%$ resistant to eight antibiotics; $14.7 \%$ resistant to nine antibiotics and $8.8 \%$ isolates resistant to ten antibiotics out of eleven antibiotics. This shows that most of the isolates were resistant to seven and eight antibiotics among the antibiotics tested which indicated that there were high numbers of multidrug-resistant bacteria being eluted due to the used of the multiple drugs in livestock farming resulting in the development of antimicrobial resistant bacteria infestation. Lowy (2003) mentioned that $S$. aureus became increasingly resistant towards a greater number of the antimicrobial agent when it comes to a sample from the blood culture of worldwide and intensive care units country. This is highly related to this study as the untreated wastewater collected consists of blood and high organic content, categorized as a high degree of contamination.

The release of high antimicrobial resistance of $S$. aureus via the wastewater creates a reservoir of antibiotic resistant microorganisms. This promotes the genes transfer from one microorganism to another microorganism through vertical or horizontal transfer. Genes transfer can also occur through bacteriophage. It was reported that mecA gene encoded for the methicillin resistance in MRSA was found in the DNA of the bacteriophage (Colomer-Lluch et al., 2011; Berglund, 2014) through the transduction process. Bacteriophage acts as vehicles for antibiotic resistance genes. The uptake of MRSA by the lytic-type bacteriophage could have mediated the acquisition of mecA gene. This has been demonstrated in clinically relevant bacterial species as reported by Balcazar (2014). In addition, marine living organisms, plants and the environment are also affected if the wastewater is untreated properly. A study reported that the whales' lungs were heavily colonized with antimicrobial resistance bacteria (Raverty et al., 2017). The cycle, however, does not stop there. Human consumes fish and seafood products as food; hence, the bacteria will coincidently be transferred back to the humans. In the U.S., it was reported that S. aureus was among the top five pathogens with approximately 241,148 cases annually (Abdalrahman et al., 2015) and it is one of the most important causes of gastroenteritis worldwide (Jamshidi and Palsson, 2008). This route of exposure to high antimicrobial resistance $S$. aureus to humans could occur in this manner. Similarly, this was observed in this study. Livestock is heavily colonized 
with antimicrobial resistant bacteria and humans consume the poultry in which eventually, they contract the antimicrobial resistant bacteria. Derived food products could also possibly have or contaminate with the residual antimicrobial resistant bacteria once the bacteria gain entry into the food supply chain, thus, increasing the chances of dissemination (Garofolo et al., 2007). The infection by antimicrobial resistance leads to serious consequences especially to a sickened individual which results in an increase in the number of victims, severity, and duration of infections (Rogers and Haines, 2005).

Baddour et al. (2016) stressed on the need for the treatment of wastewater produced by technical poultry slaughterhouse as it is not practiced globally. This is supported by Rogers and Haines (2005) which mentioned that several researchers had identified the highest level of antimicrobial resistant bacteria in animal farmers and high in slaughterhouse workers. The movement is from the farm animal to farmer and slaughterer. The potential risk of infection by contaminated livestock waste depends on the routes, the dose, the responsible agent and the exposed individual themselves (Rogers and Haines, 2005). As the issue exacerbates year by year, the respective organizations need to be more particular to solve this issue.

If resistant bacteria are transmitted to humans, antibiotic against the pathogen infection will no longer be effective. This calls for the need of new strategies to control the spread of the antimicrobial resistance bacteria (Ghafouryan, 2014). According to Lowy (2003), the currently available antimicrobial agents is quinupristindalfopristin, which is protein synthesis inhibitor together with gram-positive spectrum to protect against drugresistant Staphylococci. However, the drugs are still ongoing clinical trials. In addition, Kumar et al. (2015) suggested the need for organizing health and hygienerelated programs to the public as to raise the awareness related to $S$. aureus which emphasizes on sanitary and drug-related guidelines.

Despite that, the country's economy also suffers greatly if no preventive measures are taken. Once foodborne illness occurs in a certain state or country, the medical cost of expenditure will be high. For example, there was a Milwaukee outbreak of Cryptosporidiosis mentioned by Rogers and Haines (2005) in 1993 found that in community cost as much as 96.2 million; 31.7 million in medical costs and 64.6 million in lost productivity (Harrison and Lederberg, 1998; Corso et al., 2003). For a more severe case, one person can cost up to $\$ 9,500$ (Rogers and Haines, 2005). Consequently, it is important to overcome this issue as to reduce the treatment costs especially for owners of industrial plants and to preserve the environment (Baddour et al., 2016). Further study needs to be done in order to save the environment as well as human, economic and others.

\section{Conclusion}

In conclusion, all samples (100\%) are positive for $S$. aureus which ranged between 11 and $21000 \mathrm{MPN} / \mathrm{ml}$. The originating point of the $S$. aureus could be from the infested livestock, slaughterhouse, and food handlers. Isolated $S$. aureus showed a high percentage of resistance towards amoxicillin, ampicillin, and penicillin and $94.1 \%$ of the strains can be categorized as MRSA. The release of the high antimicrobial resistant $S$. aureus through untreated wastewater from the slaughterhouse to the environment will pose a serious threat to humans. Hence, drastic actions should be implemented with effective monitoring and control to ensure the wastewater should be treated before release to the environment. Additionally, surveillance of antimicrobial resistance bacteria, especially MRSA, should also be conducted regularly for monitoring and data collection. Most importantly, guidelines and recommendations on the use of antibiotic, the types, the dose and the frequency should be established as an act to control the antimicrobial resistant bacteria.

\section{Conflict of Interest}

The authors declare no conflict of interest

\section{Acknowledgement}

Research fund was sponsored by the Universiti Putra Malaysia Grant GP-IPS 2015 (GP-IPS/2015/9466100). The authors would like to express their gratitude to the poultry slaughterhouse in Semenyih, Selangor for their consent to perform the research project.

\section{References}

Abdalrahman, L.S., Stanley, A., Wells, H. and Fakhr, M.K. (2015). Isolation, Virulence, and Antimicrobial Resistance of Methicillin-Resistant Staphylococcus aureus (MRSA) and Methicillin Sensitive Staphylococcus aureus (MSSA) Strains from Oklahoma Retail Poultry Meats. International Journal of Environmental Research and Public Health, 12(6), 6148-6161. https://doi.org/10.3390/ ijerph120606148

Appelbaum, P.C. (2012). 2012 and beyond: potential or the start of a second pre-antibiotic era? The Journal of Antimicrobial Chemotherapy, 67(9), 2062-2068. 
https://doi.org/10.1093/jac/dks213

Arai, T., Okada, S. and Shimizu, A. (2004). Staphylococcus aureus contamination in a poultryprocessing plant and biological properties of isolates. Journal of the Japan Veterinary Medical Association 57: 460-464.United Nations. 2017. The United Nations World Water Development Report 2017. Wastewater. The Untapped Resource. France: the United Nations Educational, Scientific and Cultural Organization.

Baddour E. M., Farhoud N., Sharholy M. and AbdelMagid I. M. (2016). Biological Treatment of Poultry Slaughterhouses Wastewater by Using Aerobic Moving Bed Biofilm Reactor. International Research Journal of Public and Environmental Health, 3(5), 96-106.

Balcazar, J.L. (2014). Bacteriophages as Vehicles for Antibiotic Resistance Genes in the Environment. PLoS Pathogens, 10(7), e1004219. https:// doi.org/10.1371/journal.ppat.1004219

Bauer, A.W., Kirby, W.M., Sherris, J.C. and Turck, M. (1966). Antibiotic susceptibility testing by a standardized single disk method. American Journal of Clinical Pathology, 45(4), 493-496.

Bennett, R.W. and Lancette, G.A. (2016). Bacteriological Analytical Manual Chapter 12. Staphylococcus aureus. Retrieved on December 29, 2017 from U.S. Food and Drug Administration website: https://www.fda.gov/Food/ FoodScienceResearch/LaboratoryMethods/ ucm071429.htm

Berglund, B. (2014). Deliberations on The Impact of Antibiotic Contamination on dissemination of antibiotic resistance genes in aquatic environments. Retreived from Linkoping University: https:// pdfs.semanticscholar.org/

f8d5/6df44c838a69e387b6200920e604448d08ce.pdf

Bhatia, R. and Narain, J.P. (2010). The growing challenge of antimicrobial resistance in the SouthEast Asia Region - Are we losing the battle? Indian Journal of Medical Research, 132(5), 482-486. https: doi.org/10.4103/0971-5916.73313

Blodgett, R. (2015). BAM Appendix 2: Most Probable Number from Serial Dilutions. Retrieved on December 29, 2017 from U.S. Food and Drug Administration website: https://www.fda.gov/Food/ FoodScienceResearch/LaboratoryMethods/ ucm109656.htm

Brakstad, O.G., Aasbakk, K. and Maeland, J.A. (1992). Detection of Staphylococcus aureus by polymerase chain reaction amplification of the nuc gene. Journal of Clinical Microbiology, 30(7), 1654-1660
Centers for Disease Control and Prevention (CDC), (September 19, 2017). About Antibiotic/ Antimicrobial Resistance. Retrieved on December 29, 2017 from CDC website: https://www.cdc.gov/ drugresistance/about.html

Centers for Disease Control and Prevention (CDC). (2013). Antibiotic resistance threats in the United States, 2013. US: Department of Health and Human Services Centers for Disease Control and prevention.

Centers for Disease Control and Prevention (CDC). (2016). Methicillin-resistant Staphylococcus aureus (MRSA), Laboratory Testing for MRSA. Retrieved on June 9, 2016 from CDC website: https:// www.cdc.gov/mrsa/lab/index.html

Clinical and Laboratory Standards Institute (CLSI). (2016). M100S Performance Standards for Antimicrobial Susceptibility Testing. $26^{\text {th }}$ ed. USA: CLSI.

Colomer-Lluch, M., Imamovic, L., Jofre, J. and Muniesa, M. (2011). Bacteriophages carrying antibiotic resistance genes in fecal wastes from cattle, pigs and poultry. Antimicrobial Agents and Chemotherapy, 55 (10), 4908-4911. https://doi.org/10.1128/AAC.00535 $-11$

Corso, P.S., Kramer, M.H., Blair, K.A., Addis, D.G., Davis, J.P. and Haddix, A.C. (2003). Cost of illness in the 1993 waterborne Cryptosporidium outbreak, Milwaukee, Wisconsin. Emerging Infectious Diseases, 9(4), 426-431. https://doi.org/10.3201/ eid0904.020417

DW. (2017). The business of wastewater. Retrieved on June 9, 2017 from DW website: http://www.dw.com/ en/the-business-of-wastewater/a-38061683

Food Standards Agency. (2017). Risk Assessment on Meticillin-Resistant Staphylococcus aureus (MRSA), with a focus on Livestock-associated MRSA, in the UK Food Chain. Retrieved from

Garofalo, C., Vignaroli, C., Zandri, G., Aquilanti, L., Bordoni, D., Osimani, A., Clementi, F. and Biavasco, F. (2007). Direct detection of antibiotic resistance genes in specimens of chicken and pork meat. International Journal of Food Microbiology, 113(1), 113:75-83 https://doi.org/10.1016/ j.ijfoodmicro.2006.07.015

Ghafouryan, S. (2014). Toxin Antitoxin System as An Antimicrobial Target for Antibiotic Resistant Staphylococcus aureus. Selangor, Malaysia: Universiti Putra Malaysia, Doctorate Thesis.

Harrison, P.F. and Lederberg, J. (1998). Antimicrobial resistance: issues and options workshop report. US: National Institutes of Health

Ike, B., Ugwu, M.C., Ikegbunam, M.N., Nwobodo, D., 
Ejikeugwu, C., Gugu, T. and Esimone, C.O. (2016). Prevalence, Antibiogram and Molecular Characterization of Comunity-Acquired MethicillinResistant Staphylococcus aureus in AWKA, Anambra Nigeria. The Open Microbiology Journal, 10, 211-221. https:// doi.org/10.2174/1874285801610010211

Jamshidi, N. and Palsson, B.O. (2008). Formulating genome-scale kinetic models in the post-genome era. Molecular Systems Biology, 4(1), 171. https:// doi.org/10.1038/msb.2008.8

Kluytmans, J.A. (2010). Methicillin-resistant Staphylococcus aureus in food products: cause for concern or case for complacency? Clinical Microbiology and Infection: the official publication of the European Society of Clinical Microbiology and Infectious Disease, 16(1), 11-15

Kumar, H., Palaha, R., Kaur, N. and Ratnakar, W.S. (2015). Prevalence of multidrug-resistant, coagulasepositive Staphylococcus aureus in nasal carriage, food, wastewater and paper currency in Jalandhar city (north-western), an Indian state of Punjab. Environmental Monitoring and Assessment, 187, 4134. https://doi.org/10.1007/s10661-014-4134-6

Landers, T.F., Cohen, B., Wittum, T.E. and Larson, E.L. (2012). A review of antibiotic use in food animals: perspective, policy and potential. Public Health Reports, 127(1), 4 - 22. https://doi.org/ $10.1177 / 003335491212700103$

Losito, P., Vergara, A., Muscariello, T. and Ianieri, A. (2005). Antimicrobial Susceptibility of Environmental Staphylococcus aureus Strains Isolated from a Pigeon Slaughterhouse in Italy. Poultry Science, 84, 1802-1807. https:// doi.org/10.1093/ps/84.11.1802

Lowy, F. 2003. Antimicrobial Resistance: The Example of Staphylococcus aureus. Journal of Clinical Investigation, 111(9), 1265-1273. https:// doi.org/10.1172/JCI18535

Ministry of Health, Malaysia. (2006). Food Consumption Statistics of Malaysia 2003. Malaysia: Family Health Development Division, Food Safety and Quality Division, Department of Public Health, Ministry of Health Malaysia.

New, C.Y., Amalia, A.R., Ramzi, O.S.B. and Son, R. (2016). Antibiotic resistance evolution of Methicillin Resistant Staphylococcus aureus (MRSA) and colloidal silver as the nanoweapon. International Food Research Journal, 23(3), 1248-1254

Rahman, N.A., Jalal, A., Chowdhury, K. and Azira, Z. (2015). Antibiotic Resistant Bacteria from Sediment of Coastal Water of Pahang, Malaysia. Jurnal
Teknologi (Sciences and Engineering), 24(77), 6570. https://doi.org/10.11113/jt.v77.6709

Raverty, S.A., Rhodes, L.D., Zabek, E., Eshghi, A., Cameron, C.E., Hanson, M.B. and Schroeder, J.P. 2017. Respiratory Microbiome of Endangered Southern Resident Killer Whales and Microbiota of Surrounding Sea Surface Microlayer in the Eastern North Pacific. Scientific Reports, 7. https:// doi.org/10.1038/s41598-017-00457-5

Rogers, S. and Haines, J. (2005). Detecting and Mitigating the Environmental Impact of Fecal Pathogens Originating from Confined Animal Feeding Operations: Review, p. 1-175. USA: Environmental Protection Agency

Rowan, N.J. (2011). Defining Established and Emerging Microbial Risks in the Aquatic Environment: Current Knowledge, Implications, and Outlooks. International Journal of Microbiology, 2011, 15. https://doi.org/10.1155/2011/462832

Sarmah, A.K., Meyer, M.T. and Boxall, A.B. (2006). A global perspective on the use, sales, exposure pathways, occurrence, fate and effects of veterinary antibiotics (VAs) in the environment. Chemosphere, 65, 725-759. https://doi.org/10.1016/ j.chemosphere.2006.03.026

Sutton, S. (2010). The Most Probable Number Method and Its Uses in Enumeration, Qualification, and Validation. Journal of Validation Technology, 16(3), 35-38.

The World Bank (2017). Malaysia. Retrieved on December 29, 2017 from World Bank website: https://data.worldbank.org/country/malaysia? view $=$ chart

Wright, G.D. (2010). Q and A: Antibiotic resistance: where does it come from and what can we do about it? BMC Biology, 8, 123. https:// doi.org/10.1186/1741-7007-8-123 